\title{
Prática de atividade física e fisioterapia em indivíduos com doença de Parkinson
}

\section{The practice of physical activity and physical therapy in individuals with Parkinson's disease}

(D) Alisson Luis Silva Alvim¹, (D) Lara de Almeida Rodrigues ${ }^{1}$, (D) Aline Gonçalves Gomes ${ }^{2}$, (D) Paulo Pereira Christo ${ }^{3}$, (Drancisco Eduardo Cardoso ${ }^{3}$, DPaula Luciana Scalzo ${ }^{4}$

\footnotetext{
${ }^{1}$ Discente de Fisioterapia, Universidade Federa de Minas Gerais - UFMG

2 Doutoranda, Programa de Pós-Graduação em Neurociências da Universidade Federal de Minas Gerais - UFMG

3 Professor, Faculdade de Medicina da Universidade Federal de Minas Gerais - UFMG

4 Professora Associada, Departamento de Morfologia do Instituto de Ciências Biológicas; Programa de Pós-Graduação em Neurociências da Universidade Federal de Minas Gerais UFMG
}

Correspondência

Paula Luciana Scalzo

E-mail: paula@icb.ufmg.br

Submetido: 24 Abril 2020

Aceito: 21 Outubro 2020

Como citar

Alvim ALS, Rodrigues LA, Gomes AG, Christo PP, Cardoso FE, Scalzo PL. Prática de atividade física e fisioterapia em indivíduos com doença de Parkinson. Acta Fisiatr. 2020;27(3):146-151.

DOI: 10.11606/issn.2317-0190.v27i3a169050

\section{cc) $(1)$}

\section{RESUMO}

Objetivo: Avaliar o perfil da prática de atividade física (AF) e de fisioterapia em indivíduos com doença de Parkinson (DP). Métodos: Pacientes foram recrutados a partir de dois centros de desordens de movimento de Belo Horizonte (Ambulatório Bias Fortes da Universidade Federal de Minas Gerais e Centro Metropolitano de Especialidades Médicas da Santa Casa de Belo Horizonte) entre fevereiro a dezembro de 2019. Resultados: Cento e oitenta e cinco indivíduos responderam um questionário para coleta de dados sociodemográficos, história médica e prática de AF e/ou fisioterapia. Ao comparar os indivíduos dos dois centros, houve diferença apenas em relação à frequência de comorbidades. A hipertensão arterial foi a comorbidade mais comum. Apenas $37,8 \%$ e $20,5 \%$ dos indivíduos relataram praticar AF ou fazer fisioterapia, respectivamente. A caminhada foi a atividade física mais comum. Pacientes sedentários tinham idade mais avançada, menor tempo de escolaridade, maior duração da DP, e eram mais acometidos por outras comorbidades quando comparados aos pacientes mais ativos. Conclusão: $O$ presente estudo mostra a influência da idade, da escolaridade e do tempo de DP na adesão à prática de AF e fisioterapia. Iniciativas de saúde pública são necessárias para promover a mudança de comportamento e melhorar as oportunidades de AF entre os indivíduos com DP.

Palavras-chave: Doença de Parkinson, Atividade Motora, Modalidades de Fisioterapia

\begin{abstract}
Objective: The present study aimed to evaluate the profile of physical activity (PA) and physical therapy in individuals with Parkinson's disease (PD). Methods: Patients were recruited from two Outpatient Movement Disorder Clinics (Ambulatório Bias Fortes, Universidade Federal de Minas Gerais, and Centro Metropolitano de Especialidades Médicas, Santa Casa de Belo Horizonte) between February and December 2019. Results: One hundred and eighty-five individuals answered a questionnaire with sociodemographic profiles, medical conditions, and the practice of PA and physical therapy. When comparing individuals from the two centers, differences were only observed concerning the frequency of comorbidities. Arterial hypertension proved to be the most prevalent comorbidity. Only $37.8 \%$ and $20.5 \%$ of the individuals reported practicing PA or physical therapy, respectively. Walking was the most common physical activity. Sedentary patients were older, had lower levels of education, presented a longer duration of PD, and were more affected by other comorbidities, when compared to more physically active patients. Conclusion: The present study shows the influence of age, education, and PD time on adherence to the practice of PA and physical therapy. Public health initiatives are needed to promote behavioral change and improve PA opportunities among individuals with PD.
\end{abstract}

Keywords: Parkinson Disease, Motor Activity, Physical Therapy Modalities 


\section{INTRODUÇÃO}

A atividade física (AF) pode ser definida como qualquer movimento corporal produzido pelos músculos esqueléticos que resulta em gasto energético ${ }^{1}$ e pode ser dividida em $A F$ estruturada e não estruturada. ${ }^{2} \mathrm{~A} A \mathrm{~F}$ estruturada corresponde ao exercício físico planejado e que visa melhorar ou manter a aptidão física. ${ }^{1}$ Já a AF não estruturada pode ser exemplificada por atividades habituais como ir ao trabalho, atividades domésticas, dança, entre outras. ${ }^{2}$

No contexto clínico, os níveis diários de AF também podem ser influenciados por programas de intervenção terapêutica não farmacológica. ${ }^{3}$ A fisioterapia é um exemplo de intervenção, centrada no paciente, que visa restaurar e maximizar a independência funcional, e que comumente utiliza o exercício físico. ${ }^{3,4} \mathrm{Na}$ doença de Parkinson (DP), a fisioterapia é principalmente uma intervenção baseada em exercícios que abordam a aptidão física, transferências, atividades manuais, equilíbrio e marcha. ${ }^{3,4}$

A AF tem sido proposta como um fator de prevenção e modificação da DP., 5 , No entanto, mais pesquisas são necessárias para investigar a influência de fatores de estilo de vida na progressão da doença. ${ }^{7}$ Estudos clínicos apontam que a sua prática, principalmente o exercício físico aeróbico, ${ }^{8}$ exerce efeitos positivos como a melhora da marcha e aptidão cardiorrespiratória ${ }^{9}$, desempenho motor ${ }^{10}$ e função cognitiva. ${ }^{11}$ No entanto, os mecanismos subjacentes a esses efeitos são pouco elucidados.

Algumas hipóteses são o aumento do fluxo sanguíneo cerebral, aumento da disponibilidade de neurotransmissores, redução da resposta inflamatória e aumento de fatores que promovem a neuroplasticidade. ${ }^{12,13}$ Esses fatores estão envolvidos com muitas funções no sistema nervoso, como remodelamento dendrítico, crescimento axonal, plasticidade sináptica e sobrevivência neuronal, inclusive em áreas motoras como os núcleos da base, ${ }^{14}$ podendo ativar cascatas antiapoptóticas e promover a sobrevivência de neurônios dopaminérgicos. ${ }^{15}$

Considerando que estudos mostram os efeitos benéficos e modificadores da prática da $A F$ na $D P,{ }^{8-11}$ a melhora de oportunidades para a sua prática poderia resultar em mudança de comportamento e diminuição do sedentarismo nessa população. Com isso, o conhecimento acerca do padrão de atividades desses indivíduos pode nortear condutas terapêuticas e iniciativas de saúde pública.

\section{OBJETIVO}

Avaliar o perfil da prática de AF de indivíduos com diagnóstico de DP idiopática que fazem acompanhamento clínico no Ambulatório Bias Fortes (ABF) anexo do Hospital das Clínicas da Universidade Federal de Minas Gerais e no Centro Metropolitano de Especialidades Médicas (CEM) da Santa Casa de Belo Horizonte.

\section{MÉTODO}

Trata-se de um estudo exploratório-descritivo que faz parte de uma pesquisa mais ampla com o objetivo de avaliar o efeito de um treino aeróbico em parâmetros biológicos e clínicos em indivíduos com DP e aprovado pelo Comitê de Ética em Pesquisa da Universidade Federal de Minas Gerais e da Santa Casa de Belo Horizonte (CAAE: 00857318.3.0000.5149).

Foram recrutados indivíduos com DP idiopática, com capacidade de entender as perguntas do questionário e em acompanhamento clínico no $A B F$ e no CEM, no período de fevereiro a dezembro de 2019. Durante essa etapa, foi aplicado um questionário para coleta de dados sociodemográficos, clínicos e relacionados à prática de $\mathrm{AF}$.

As variáveis foram: perfil dos entrevistados, tempo de sintomas e de diagnóstico da DP, uso de levodopa, prática de AF (se pratica, tipo e frequência) e fisioterapia, presença de outras doenças e necessidade de cuidador. Indivíduos com outras doenças neurológicas e psiquiátricas foram excluídos.

A análise estatística foi realizada usando o programa estatístico Statistical Package for the Social Sciences (SPSS), versão 21.0, e foi considerado $p<0,05$ como nível de significância. Foi utilizada a estatística descritiva para apresentação das variáveis.

O teste de Shapiro-Wilk foi utilizado para a análise de normalidade dos dados. O teste do Qui-Quadrado foi utilizado para comparar variáveis categóricas. Os testes T para amostras independentes e Teste de Mann-Whitney foram utilizados para comparar variáveis quantitativas, de acordo com a sua distribuição.

\section{RESULTADOS}

Cento e oitenta e cinco indivíduos responderam o questionário, sendo que 107 (57,8\%) estavam em acompanhamento clínico no ABF e 78 (42,2\%) no CEM. Desses, a maioria era do sexo masculino $(106,57,3 \%)$, tinham acima de 60 anos $(118,63,8 \%)$ e com baixa escolaridade (71 indivíduos, $38,4 \%$, tinham até 4 anos de estudo). Os dados sociodemográficos e clínicos dos participantes estão representados (Tabela 1 ).

Observa-se que houve diferença estatisticamente apenas em relação à frequência de comorbidades quando comparando os indivíduos dos dois centros de atendimento. A distribuição dos indivíduos que fazem acompanhamento no ABF e no CEM quanto à região em que reside está ilustrada (Figura 1).

Dos 185 participantes do estudo, 63 (34,1\%) praticavam AF, 31 (16,8\%) faziam fisioterapia e 7 (3,8\%) faziam ambas as atividades (Figura 2). Não houve diferença estatisticamente significativa em relação à frequência de indivíduos ativos e sedentários ao comparar os dois centros de referência ( $p=$ 0,148 ), representados na (Tabela 2 ).

Observa-se que os indivíduos que não praticavam $\mathrm{AF}$ e/ou fisioterapia tinham idade mais avançada, menor tempo de escolaridade, maior tempo de duração dos sintomas e de diagnóstico da DP e eram mais acometidos por outras comorbidades. As informações quanto à modalidade de AF praticada pelos participantes estão representadas na Figura 3.

Em relação à frequência da $\mathrm{AF}, 5(7,1 \%)$ indivíduos praticavam a atividade apenas uma vez por semana, $18(25,7 \%)$ duas vezes por semana, $15(21,4 \%)$ três vezes por semana, 4 $(5,7 \%)$ quatro vezes por semana, $17(24,3 \%)$ cinco vezes por semana e $9(12,9 \%)$ sete vezes por semana e $2(2,9 \%)$ não declararam. Em relação à fisioterapia, a maioria 16 (42,1\%) realizava duas sessões por semana, $10(26,3 \%)$ três vezes por 
semana e $9(23,7 \%)$ apenas uma vez por semana. Apenas um $(2,6 \%)$ participante realizava sessões quatro e outro $(2,6 \%)$,

cinco vezes por semana. Um (2,6\%) participante não relatou a frequência das sessões de fisioterapia.

Tabela 1. Dados sociodemográficos e clínicos dos participantes do estudo

\begin{tabular}{|c|c|c|c|c|}
\hline Variáveis & $\begin{array}{l}\text { Participantes } \\
(n=185)\end{array}$ & $\begin{array}{c}\text { ABF } \\
(n=107)\end{array}$ & $\begin{array}{c}\text { CEM } \\
(n=78)\end{array}$ & Valor de $p$ \\
\hline Idade (anos) & $64,1( \pm 11,2)$ & $64,9( \pm 11,9)$ & $63( \pm 10,1)$ & 0,251 \\
\hline Sexo (homem/mulher), $\mathrm{n}$ & $106 / 79$ & $61 / 46$ & 45 / 33 & $0,926 *$ \\
\hline Tempo Escolaridade (anos) & $6,7( \pm 3,9)$ & $6,2 \pm 3,7$ & $7,3 \pm 4,1$ & 0,061 \\
\hline Possui cônjuge (sim), n(\%) & $108(58,4 \%)$ & $58(54,2 \%)$ & $50(64,1 \%)$ & $0,177^{*}$ \\
\hline \multicolumn{5}{|l|}{ Reside em, $n(\%)$} \\
\hline Mora com a família & $170(91,9 \%)$ & $97(90,7 \%)$ & $73(93,6 \%)$ & \\
\hline Mora em lar de acolhimento & $2(1,1 \%)$ & $2(1,9 \%)$ & - & $0,629 \#$ \\
\hline Mora sozinho & $13(7 \%)$ & $8(7,5 \%)$ & $5(6,4 \%)$ & \\
\hline \multicolumn{5}{|l|}{ Ocupação, n(\%) } \\
\hline Afastado/Desempregado & $16(8,7 \%)$ & $10(9,4 \%)$ & $6(7,7 \%)$ & \\
\hline Aposentado & $161(87 \%)$ & $90(84,1 \%)$ & $71(91,0 \%)$ & $0,234^{\#}$ \\
\hline Ativo & $8(4,3 \%)$ & $7(6,5 \%)$ & $1(1,3 \%)$ & \\
\hline Tempo Sintomas DP (anos) & $11,9( \pm 6,7)$ & $12,4 \pm 7,0$ & $10,9 \pm 5,9$ & 0,219 \\
\hline Tempo Diagnóstico DP (anos) & $10,6( \pm 6,5)$ & $11,3 \pm 7,1$ & $9,2 \pm 5,0$ & 0,058 \\
\hline Uso de levodopa (sim), $\mathrm{n}(\%)$ & $183(99 \%)$ & $105(98,1 \%)$ & $78(100 \%)$ & $0,510^{\#}$ \\
\hline Doenças associadas (sim), $n(\%)$ & $103(55,7 \%)$ & $67(62,6 \%)$ & $36(46,2 \%)$ & $0,026 *$ \\
\hline Ansiedade/Depressão & $12(6,5 \%)$ & $9(8,4 \%)$ & $3(3,8 \%)$ & \\
\hline Diabetes & $16(8,6 \%)$ & $12(11,2 \%)$ & $4(5,1 \%)$ & \\
\hline Dislipidemia & $3(1,6 \%)$ & $3(2,8 \%)$ & - & \\
\hline Doenças Cardíacas & $4(2,2 \%)$ & $3(2,8 \%)$ & $1(1,3 \%)$ & \\
\hline Doenças Osteomusculares & $23(12,4 \%)$ & $15(22,4 \%)$ & $8(10,3 \%)$ & \\
\hline HAS & $59(31,9 \%)$ & $38(35,5 \%)$ & $21(26,9 \%)$ & \\
\hline Hipotireoidismo & $10(5,4 \%)$ & $7(6,5 \%)$ & $3(3,8 \%)$ & \\
\hline Outras & $26(14,1 \%)$ & $16(15 \%)$ & $10(12,8 \%)$ & \\
\hline Cuidador (sim), n(\%) & $55(29,7 \%)$ & $34(31,8 \%)$ & $21(26,9 \%)$ & $0,476 *$ \\
\hline
\end{tabular}

ABF= Ambulatório Bias Fortes; CEM= Centro de Especialidades Médicas da Santa Casa de Belo Horizonte; HAS= Hipertensão Arterial Sistêmica; AF= Atividade Física. Teste t para amostras independentes para comparação de variáveis quantitativas entre grupos. Para variáveis categóricas: "Teste Exato de Fisher ou *Teste do Qui-Quadrado para outras variáveis

Tabela 2. Características dos participantes que praticam e não praticam atividade física e/ou fisioterapia

\begin{tabular}{|c|c|c|c|}
\hline Variáveis & $\begin{array}{l}\text { Pratica AF e/ou fisioterapia } \\
(n=101)\end{array}$ & $\begin{array}{l}\text { Não pratica AF e/ou } \\
\text { fisioterapia }(n=84)\end{array}$ & Valor de $p$ \\
\hline Idade (anos) & $61,6( \pm 11,2)$ & $67,2( \pm 10,5)$ & 0,002 \\
\hline Sexo (homem / mulher) & $58 / 43$ & $48 / 36$ & $0,834^{*}$ \\
\hline Tempo Escolaridade (anos) & $7,4( \pm 4,0)$ & $5,8( \pm 3,7)$ & 0,007 \\
\hline Possui cônjuge (sim), n (\%) & $65(64,3 \%)$ & $43(51,2 \%)$ & $0,092 *$ \\
\hline \multicolumn{4}{|l|}{ Reside em, $n(\%)$} \\
\hline Mora com a família & $94(93,1 \%)$ & $76(90,5 \%)$ & \multirow{3}{*}{$0,789^{\#}$} \\
\hline Mora em lar de acolhimento & $1(1 \%)$ & $1(1,2 \%)$ & \\
\hline Mora sozinho & $6(5,9 \%)$ & $7(8,3 \%)$ & \\
\hline \multicolumn{4}{|l|}{ Ocupação, n (\%) } \\
\hline Afastado/Desempregado & $12(11,9 \%)$ & $4(4,8 \%)$ & \multirow[t]{3}{*}{$0,148^{\#}$} \\
\hline Aposentado & $86(85,1 \%)$ & $75(89,3 \%)$ & \\
\hline Ativo & $3(3 \%)$ & $5(5,9 \%)$ & \\
\hline Tempo Sintomas DP (anos) & $10,9( \pm 6,1)$ & $13,0( \pm 7,2)$ & 0,035 \\
\hline Tempo Diagnóstico DP (anos) & $9,6( \pm 5,6)$ & $11,7( \pm 7,4)$ & 0,041 \\
\hline Uso de levodopa (sim), n (\%) & $101(100 \%)$ & $82(97,6 \%)$ & $0,210^{\#}$ \\
\hline Doenças associadas (sim), n (\%) & $45(44,6 \%)$ & $58(69 \%)$ & \multirow{8}{*}{$0,001 *$} \\
\hline Ansiedade/Depressão & $5(4,9 \%)$ & $7(8,3 \%)$ & \\
\hline Diabetes & $8(7,9 \%)$ & $8(9,5 \%)$ & \\
\hline Dislipidemia & $2(2 \%)$ & $1(1,2 \%)$ & \\
\hline Doenças Cardíacas & $1(1 \%)$ & $3(3,6 \%)$ & \\
\hline Doenças osteomusculares & $13(12,9 \%)$ & $10(11,9 \%)$ & \\
\hline HAS & $25(24,8 \%)$ & $34(40,5 \%)$ & \\
\hline Hipotireoidismo & $3(3 \%)$ & $7(8,3 \%)$ & \\
\hline Necessidade de cuidador (sim), n (\%) & $25(24,8 \%)$ & $30(35,7 \%)$ & $0,127 *$ \\
\hline
\end{tabular}

AF= Atividade Física; HAS= Hipertensão Arterial Sistêmica; $A F=$ Atividade Física. Teste t para amostras independentes para comparação de variáveis quantitativas entre grupos. Para variáveis, categóricas: \#Teste Exato de Fisher ou *Teste do Qui-Quadrado para outras variáveis 
Distribuição dos Indivíduos por Região

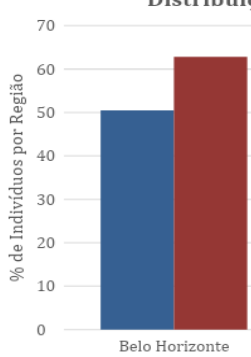

Belo Horizonte
- $\mathrm{ABF}$ - $\mathrm{CEM}$

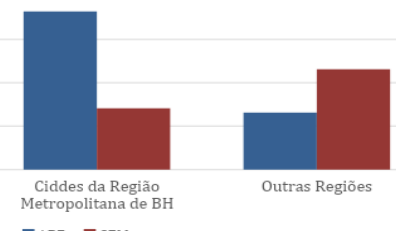

Figura 1. Distribuição dos indivíduos de acordo com a região em que reside

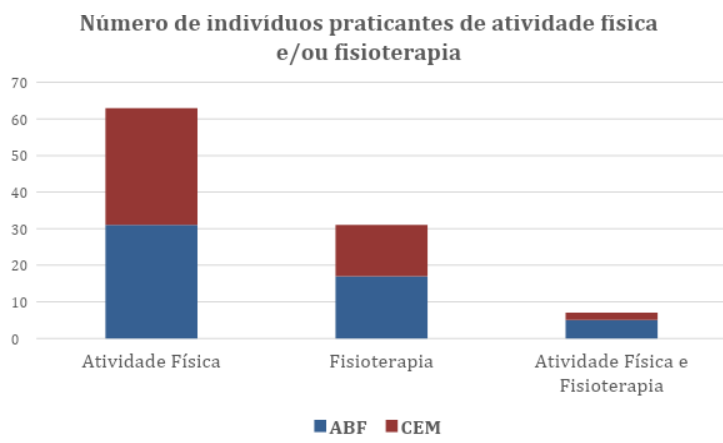

Figura 2. Número de indivíduos que fazem atividade física e/ou fisioterapia

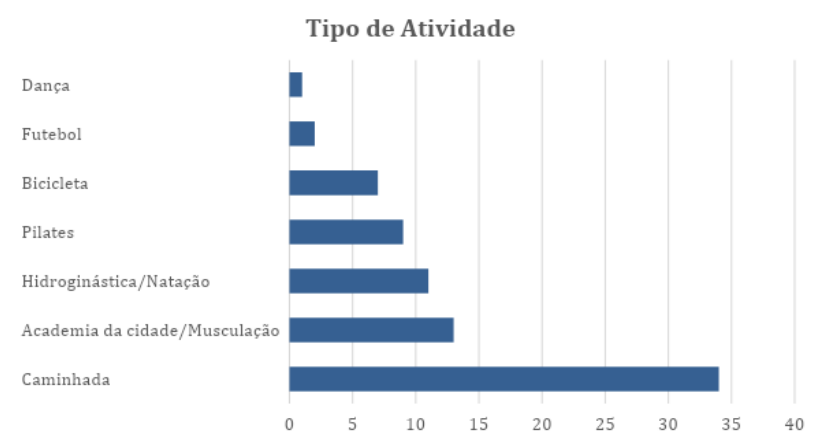

Figura 3. Tipo de atividade física realizada pelos participantes do estudo

\section{DISCUSSÃO}

As características dos indivíduos com DP que fazem acompanhamento clínico nos dois centros da cidade de Belo Horizonte mostram o predomínio de idosos, do sexo masculino, casados, residindo com a família, aposentados e com baixo nível de escolaridade (Tabela 1). A principal comorbidade relatada pela maioria dos participantes foi a hipertensão arterial.

De acordo com as Figuras 2 e 3, observa-se que a prática de AF foi relatada apenas por $37,8 \%$ dos participantes, sendo a caminhada a atividade mais praticada. A fisioterapia foi relatada por apenas $20,5 \%$ dos participantes.

Conforme evidenciado na Tabela 2, ao comparar os grupos, os indivíduos que não praticavam $\mathrm{AF}$ e/ou fisioterapia tinham idade mais avançada, menor tempo de escolaridade, maior tempo de duração dos sintomas e diagnóstico da DP, e eram mais acometidos por outras comorbidades.

Esses resultados corroboram outros estudos da literatura que mostram características semelhantes dos indivíduos com
DP. A idade e o estágio da doença se apresentam como variáveis significativas ao avaliar o tempo gasto em AF. ${ }^{16}$

A duração dessas atividades tende a ser menor quando avaliados pacientes com idade mais avançada e/ou com sintomatologia mais grave da doença. ${ }^{16} \mathrm{O}$ nível de escolaridade é um fator apresentado em outros estudos que mostram que a adesão à prática de AF em idosos é menor por pessoas com menor escolaridade. ${ }^{17} \mathrm{Um}$ importante fator que deve ser considerado e pode influenciar a adesão à prática de diferentes modalidades terapêuticas é a motivação do indivíduo com DP. ${ }^{18}$

Em relação às comorbidades, a hipertensão arterial corresponde ao distúrbio cardiovascular de maior prevalência na população adulta e principalmente nos idosos no Brasil. ${ }^{19}$ É descrito que a inatividade física e o estilo de vida sedentário estão relacionados a fatores de risco para o desenvolvimento ou agravamento de condições médicas, como alterações cardiovasculares e metabólicas. ${ }^{20}$ Os programas de exercício estão associados à prevenção do desenvolvimento de hipertensão e também à redução da pressão arterial. ${ }^{20}$

Em relação à modalidade de intervenção ativa mais relatada, a caminhada é geralmente uma boa estratégia por não envolver habilidades especiais, equipamentos ou ambientes específicos. ${ }^{21}$ Além disso, com a melhora da aptidão, o aumento da duração e frequência da caminhada podem determinar uma AF de maior intensidade. ${ }^{21}$ Considerando o perfil de indivíduos que não praticavam AF e/ou fisioterapia, deve-se incentivar a redução de comportamentos sedentários, como por exemplo, atividades não relacionadas ao exercício, ${ }^{21}$ incluindo atividades domésticas e de lazer.

Muitos estudos mostram que a $A F$, especificamente quando realizada de forma estruturada e planejada sendo caracterizada como exercício físico, é capaz de induzir a neuroplasticidade a partir de alterações moleculares, estruturais, funcionais e comportamentais. ${ }^{22-24}$

Em nível molecular ocorrem mudanças nas concentrações de fatores neurotróficos como o fator neurotrófico derivado do cérebro (BDNF, do inglês brain-derived neurotrophic factor), fator de crescimento do nervo (NGF, do inglês nerve growth factor), fator de crescimento semelhante à insulina (IGF-1, do inglês insulin-like growth factor) e fator de crescimento endotelial vascular (VEGF, do inglês vascular endothelial growth factor).

Esses fatores estão envolvidos em muitas funções no sistema nervoso, como remodelamento dendrítico, crescimento axonal, plasticidade sináptica e sobrevivência neuronal. 22,23

O exercício também promove a liberação de endorfina que produz euforia e bem estar. A melhora de sintomas afetivos, como depressão e ansiedade, pode ser explicada pela ligação da endorfina em receptores opióides no córtex frontal e sistema límbico, além de interagir com outros neurotransmissores como a dopamina. ${ }^{25}$

Mudanças estruturais, como aumento da substância cinzenta em áreas do hipocampo, cerebelo, núcleos da base e diferentes regiões do córtex cerebral, podem ser consequência de processos de crescimento e proliferação neuronal (neurogênese), de células da glia (gliogênese), fortalecimento e formação de novas sinapses (sinaptogênese) e a formação de novos capilares (angiogênese). Essas alterações promovem o 
aumento da disponibilidade de neurotransmissores e do fluxo sanguíneo cerebral. ${ }^{22,23}$

Alterações funcionais podem ser indicadas pelo aumento da ativação neuronal, otimização do processamento e da conectividade neural. ${ }^{22,23}$ Isso pode resultar em alterações comportamentais com melhora do desempenho cognitivo e, possivelmente, no desempenho motor e retenção de habilidades motoras. ${ }^{22,23}$ Pode ocorrer a associação positiva entre o nível de condicionamento físico e a aprendizagem de tarefas motoras, o que pode ser mediado pela melhora das funções cognitivas como atenção. ${ }^{22,23}$

Em consonância a esses resultados, revisões sistemáticas apontam que diferentes protocolos de intervenções, como caminhada na esteira e no solo, treino de resistência, dentre outros, podem promover melhora de alterações não motoras da DP, por exemplo a depressão, os distúrbios de sono, a fadiga, a apatia e o prejuízo cognitivo. ${ }^{11,26}$

A principal limitação do estudo é devido ao fato de se tratar de um estudo transversal, sem que seja possível determinar relação causal. No entanto, o estudo é importante por ter sido realizado em dois centros de referência em desordens de movimento da cidade de Belo Horizonte e de vários outros municípios da região.

Dessa forma, considerando a importância da prática de AF na DP, os resultados do presente estudo mostram a necessidade de iniciativas de saúde pública para promover a mudança de comportamento e melhorar as oportunidades para a prática de AF entre os indivíduos com DP.

\section{CONCLUSÃO}

O estudo mostra a baixa adesão à prática de $A F$ e/ou fisioterapia por indivíduos com DP que fazem acompanhamento clínico em dois centros especializados em desordens de movimento de Belo Horizonte.

Indivíduos que não realizam atividades apresentaram idade mais avançada, menor tempo de estudo, maior tempo de duração dos sintomas e do diagnóstico de DP e maior frequência de comorbidades. Dentre as atividades, a caminhada foi a mais relatada. Iniciativas de saúde pública são necessárias para promover a mudança de comportamento e melhorar as oportunidades para a prática de AF entre os indivíduos com DP.

\section{REFERÊNCIAS}

1. Caspersen CJ, Powell KE, Christenson GM. Physical activity, exercise, and physical fitness: definitions and distinctions for health-related research. Public Health Rep. 1985;100(2):126-31.

2. Levine JA. Non-exercise activity thermogenesis (NEAT). Nutr Rev. 2004;62(7 Pt 2):S82-97. Doi: https://doi.org/10.1111/i.1753-4887.2004.tb00094.x

3. Abbruzzese G, Marchese R, Avanzino L, Pelosin E. Rehabilitation for Parkinson's disease: current outlook and future challenges. Parkinsonism Relat Disord. 2016;22 Suppl 1:S60-4.

Doi:
4. Bouça-Machado R, Rosário A, Caldeira D, Castro Caldas A, Guerreiro D, Venturelli M, et al. Physical activity, exercise, and physiotherapy in parkinson's disease: defining the concepts. Mov Disord Clin Pract. 2019;7(1):7-15. Doi: https://doi.org/10.1002/mdc3.12849

5. Aaseth J, Dusek P, Roos PM. Prevention of progression in Parkinson's disease. Biometals. 2018;31(5):737-747. Doi: https://doi.org/10.1007/s10534-018-0131-5

6. Marras C, Canning CG, Goldman SM. Environment, lifestyle, and Parkinson's disease: Implications for prevention in the next decade. Mov Disord. 2019;34(6):801-811.

Doi:

https://doi.org/10.1002/mds.27720

7. Nag N, Jelinek GA. More Research Is Needed on Lifestyle Behaviors That Influence Progression of Parkinson's Disease. Front Neurol. 2019;10:452. Doi: https://doi.org/10.3389/fneur.2019.00452

8. Monteiro-Junior RS, Cevada T, Oliveira BR, Lattari E, Portugal EM, Carvalho A, et al. We need to move more: Neurobiological hypotheses of physical exercise as a treatment for Parkinson's disease. Med Hypotheses. 2015;85(5):537-41.

Doi: https://doi.org/10.1016/j.mehy.2015.07.011

9. Uhrbrand A, Stenager E, Pedersen MS, Dalgas U. Parkinson's disease and intensive exercise therapy--a systematic review and meta-analysis of randomized controlled trials. J Neurol Sci. 2015;353(1-2):9-19. Doi: https://doi.org/10.1016/i.jns.2015.04.004

10. Flach A, Jaegers L, Krieger M, Bixler E, Kelly P, Weiss EP, Ahmad SO. Endurance exercise improves function in individuals with Parkinson's disease: A meta-analysis. Neurosci Lett. 2017;659:115-119. Doi: https://doi.org/10.1016/i.neulet.2017.08.076

11. Silva FC, lop RDR, Oliveira LC, Boll AM, Alvarenga JGS, Gutierres Filho PJB, et al. Effects of physical exercise programs on cognitive function in Parkinson's disease patients: a systematic review of randomized controlled trials of the last 10 years. PLoS One. 2018;13(2):e0193113. Doi: https://doi.org/10.1371/journal.pone.0193113

12. Hirsch MA, Farley BG. Exercise and neuroplasticity in persons living with Parkinson's disease. Eur J Phys Rehabil Med. 2009;45(2):215-29.

13. Frazzitta G, Maestri R, Ghilardi MF, Riboldazzi G, Perini M, Bertotti $G$, et al. Intensive rehabilitation increases BDNF serum levels in parkinsonian patients: a randomized study. Neurorehabil Neural Repair. 2014;28(2):163-8. Doi: https://doi.org/10.1177/1545968313508474

14. He YY, Zhang XY, Yung WH, Zhu JN, Wang JJ. Role of BDNF in central motor structures and motor diseases. Mol Neurobiol. 2013;48(3):783-93. Doi: https://doi.org/10.1007/s12035-013-8466-y

15. Real CC, Ferreira AF, Chaves-Kirsten GP, Torrão AS, Pires RS, Britto LR. BDNF receptor blockade hinders the beneficial effects of exercise in a rat model of Parkinson's disease. Neuroscience. 2013;237:118-29. Doi: https://doi.org/10.1016/i.neuroscience.2013.01.060

16. van Nimwegen $M$, Speelman AD, Hofman-van Rossum EJ, Overeem S, Deeg DJ, Borm GF, et al. Physical inactivity in Parkinson's disease. J Neurol. 2011;258(12):2214-21. Doi: https://doi.org/10.1007/s00415-011-6097-7 
17. Andreotti MC, Okuma SS. Perfil sociodemográfico e perfil inicial de idosos ingressantes em um programa de Educação Física. Rev Paul Educ. Fís. 2003;17(2):142-53. Doi: https://doi.org/10.11606/issn.25945904.rpef.2003.137571

18. Gomes SR, Trippo KV, Guimarães DO, Oliveira Filho J, Dominguez-Ferraz D. Motivation of elderly with Parkinson's disease submitted to functional training, aerobic exercise and exergame. Acta Fisiatr. 2018;25(3):119-23. Doi: https://doi.org/10.11606/issn.2317-0190.v25i3a162643

19. Sociedade Brasileira de Cardiologia. 7a Diretriz Brasileira de Hipertensão Arterial. Arq Bras Cardiol. 2016;107(3 Supl.3) 1:3.

20. Pedersen BK, Saltin B. Exercise as medicine - evidence for prescribing exercise as therapy in 26 different chronic diseases. Scand J Med Sci Sports. 2015;25 Suppl 3:1-72. Doi: https://doi.org/10.1111/sms.12581

21. Piercy KL, Troiano RP, Ballard RM, Carlson SA, Fulton JE, Galuska DA, et al. The Physical Activity Guidelines for Americans. JAMA. 2018;320(19):2020-2028. Doi: https://doi.org/10.1001/jama.2018.14854
22. Phillips C, Baktir MA, Srivatsan M, Salehi A. Neuroprotective effects of physical activity on the brain: a closer look at trophic factor signaling. Front Cell Neurosci. 2014;8:170.

Doi: https://doi.org/10.3389/fncel.2014.00170

23. El-Sayes J, Harasym D, Turco CV, Locke MB, Nelson AJ. Exercise-Induced Neuroplasticity: A Mechanistic Model and Prospects for Promoting Plasticity. Neuroscientist. 2019;25(1):65-85.

Doi: https://doi.org/10.1177/1073858418771538

24. Spielman LJ, Little JP, Klegeris A. Physical activity and exercise attenuate neuroinflammation in neurological diseases. Brain Res Bull. 2016;125:19-29. Doi: https://doi.org/10.1016/j.brainresbull.2016.03.012

25. Wu PL, Lee M, Huang TT. Effectiveness of physical activity on patients with depression and Parkinson's disease: A systematic review. PLoS One. 2017;12(7):e0181515. Doi: https://doi.org/10.1371/journal.pone.0181515

26. Cusso ME, Donald KJ, Khoo TK. The Impact of Physical Activity on Non-Motor Symptoms in Parkinson's Disease: A Systematic Review. Front Med (Lausanne). 2016;3:35. Doi: https://doi.org/10.3389/fmed.2016.00035 